\title{
Desenvolvimento e Aplicação do CDA Introdução aos Estudos Virtuais
}

\author{
Vitor C. S. Valadares, Willian J. M. Fardin, João B. Mossmann, Marsal Á. A. \\ Branco, Marta R. Bez, Thiago G. Mendes \\ Laboratório de Objetos de Aprendizagem - Universidade Feevale \\ ERS-239, 2755, Novo Hamburgo - RS - Brazil \\ vitorvaladaresbr@gmail _com; willian_fardin@gmail_com; \\ mossmann@gmail_com; marsal@feevale.br; martabez@gmail.com; \\ thiagogm@feevale.br
}

\begin{abstract}
The present work has as it's objective to show the process of developing a Digital Construct of Learning created by the Laboratory of Learning Objects from the Feevale University. The article introduces authors who support the use of digital games in education and served to reinforce the methodology applied for the creation and validation of the CDA. The results were obtained through the application of the project in a group of 101 students from distance education's courses from the institution.

Resumo. O presente trabalho tem o objetivo de mostrar o processo de desenvolvimento de um construto digital de aprendizagem criado pelo Laboratório de Objetos de Aprendizagem da Universidade Feevale. O artigo introduz autores que sustentam o uso de jogos digitais na educação e que serviram para embasar a metodologia utilizada na criação e validação do construto. Os resultados foram obtidos através da aplicação do projeto em um grupo de 101 estudantes dos cursos a distância da própria instituição.
\end{abstract}

\section{Introdução}

Os jogos digitais, segundo Prensky (2001), além de entreter, podem ser utilizados para o ensino, aliando a aprendizagem séria ao entretenimento interativo. Este tipo de jogo, focado no ensino de conteúdos específicos ou voltado ao desenvolvimento de habilidades operacionais ou comportamentais, é chamado de jogo sério (MORAIS, 2009).

Diversos pesquisadores têm trabalhado na inserção de jogos como mediadores do processo de ensino e aprendizado. Alguns dedicam-se ao desenvolvimento de jogos, outros a sua aplicação e validação em sala de aula. Burguillo (2010) demonstrou como os jogos podem potencializar o aprendizado dos alunos quanto à motivação, principalmente no que tange ao despertar da curiosidade e da autonomia do aluno sobre seu próprio aprendizado. Neste é destacada uma série de fatores alicerçados por argumentos pedagógicos que dão suporte ao uso de jogos para o ensino, utilizando o exemplo de que jogos meramente recreacionais engajam o jogador em processos de pró-atividade, organização da informação, e geram soluções alternativas para a resolução de determinados problemas (Pillay, 2002). 
No final dos anos 90, a partir de um crescimento do mercado de videogames, o termo Aprendizagem Baseada em Jogos surgiu para apresentar uma alternativa ao processo de ensino tradicional, focado inteiramente no conteúdo (AN e BONK, 2009). Segundo An e Bonk (2009) esta tem a vantagem de adequar o conteúdo da era da informação em um formato viável aos nativos digitais. Tem potencial para fornecer poderosos ambientes de aprendizagem, em que o aluno pode desenvolver as habilidades essenciais da era da informação, incluindo o pensamento crítico e a capacidade de solução de problemas.

Garris, Ahlers e Driskell (2002) defendem que no uso de jogos ocorre um processo em que na entrada, o conteúdo instrucional e as características do jogo alimentam o processo de aprendizagem, que ocorre com base em julgamentos e comportamentos, e recebe feedback do sistema. O jogador se depara com uma determinada situação apresentada pelo sistema, analisa essa situação e executa uma ação. Essa ação é recebida pelo jogo, que retorna a situação modificada.

Algumas abordagens já cogitaram a utilização de games competitivos para promover o aprendizado no ensino médio e em universidades (Ebner \& Holzinger, 2007; Lawrence, 2004; Ribeiro, Simões \& Ferreira, 2009; Wallace \& Margolis, 2007).

No contexto da teoria dos jogos, o trabalho de Burguillo (2010) propõe sua utilização como suporte para uma outra abordagem onde o conhecimento é adquirido durante a competição ou a colaboração, mas o resultado do aprendizado pode ser independente da pontuação que o estudante alcançou durante o jogo.

Bourgonjon, Valcke, Soetaert e Schellens (2010), desenvolveram um estudo sobre a reflexão do uso de jogos como recurso educacional. Alguns termos já conhecidos na área da comunicação, como Digital Natives (Prensky, 2001), Net Generation (Oblinger \& Oblinger, 2005), Screenagers (Rushkoff, 1997), e Gamer Generation (Beck \& Wade, 2004), demonstram que estes jovens jamais tiveram a experiência de viver em um mundo em que não existisse a tecnologia da informação e da comunicação. Desta forma, fica evidente que estes estudantes adquiriram habilidades específicas, novas formas de pensamento e, consequentemente, diferentes preferências sobre como aprender, o que requer uma nova forma da abordagem na educação (Oblinger \& Oblinger, 2005; Prensky, 2001).

Essa geração usa o jogo para obter conhecimento em vários contextos, lhes outorgando o poder de se auto-regularem, integrando-os a diversos domínios deste campo, e fazendo-os aprender com técnicas de descobrimento dentro dos conteúdos relativos (Gee; 2003; Papert, 1980; Rieber, 1996; Watson, 2007).

Com cada vez mais pesquisadores defendendo que esta nova geração está constantemente imersa nestas novas tecnologias, destacam a necessidade de mudanças drásticas no sistema educacional. Segundo Schulmeister (2008), é preciso colocar esta imersão em uma perspectiva em que o uso das mídias reflete no desejo dos estudantes em se comunicarem, criar seus espaços dentro da sociedade e, obviamente, se distraírem através de entretenimento (Bourgonjon, Rutten, Vanhooren, Sotaert, 2008).

Para Gee (2003), mesmo que alguns estudantes tenham baixo desempenho acadêmico, fora do ambiente escolar, eles são encorajados e motivados a realizarem 
tarefas complexas, sendo um desafio, portanto, para que o sistema educacional engaje e encontre soluções que motivem os seus alunos a terem um desempenho superior na aprendizagem. Desta forma, a utilização de mecanismos que conquistem ou seduzam os alunos, como os jogos, faz-se importante, no tocante de vislumbrar um possível ganho nos índices de crescimento do aprendizado devido à utilização de tais recursos.

Para tanto, Barab, Pettyjohn, Gresalfi, Volk, Solomou (2011) apresentam um modelo de currículo que se baseia em novas formas de tecnologia para estimular os estudantes e alavancarem a absorção do conhecimento, através de um jogo online. $\mathrm{O}$ jogo conta com, aproximadamente, 55.000 estudantes, que realizam tarefas educacionais em um ambiente que depende integralmente de sua interação para a obtenção dos resultados desejados. Esta interação, que intrinsecamente está ligada às motivações que o aluno terá para realizar as tarefas, depende, também, do ambiente que o jogo proporciona, para que o jogador torne-se um agente de mudança, que entenda e assimile os conteúdos para resolver os problemas propostos em cada cenário do jogo.

Corroborando com os autores citados anteriormente, o Horizon Report 2013 (NMC, 2013), destaca a utilização de jogos digitais como uma das tecnologias importantes para o ensino, a aprendizagem e o questionamento criativo nos próximos cinco anos. Segundo o report, os jogos tem a capacidade de promover o engajamento dos alunos, enquanto jogadores, uma vez que estes proporcionam mecânicas que realizam desafios a cerca dos seus conhecimentos em uma determinada área ou problema.

Com base nos exemplos apresentados, foi desenvolvido o Construto Digital de Aprendizagem (CDA) “Introdução aos Estudos Virtuais” como uma forma de apresentar aos alunos da Universade Feevale as premissas e os aspectos essenciais do estudo a distância. O projeto é apresentado neste artigo. Na seção 2 é apresentada a metodologia de desenvolvimento do jogo. A seção 3 apresenta o jogo desenvolvido, seguido, na seção 4, de uma avaliação realizada com alunos que tiveram a experiência de uso. Por fim, as conclusões são apresentadas.

\section{Metodologia}

Em Bez et al (2012) pode ser observada uma estrutura não hierárquica utilizada para o desenvolvimento dos Contrutos Digitais de Aprendizagem, unindo esforços das quatro áreas: educação, comunicação, design e informática. O CDA "Introdução aos Estudos Virtuais” foi desenvolvido a partir dessa estrutura, unindo a pesquisa científica com 0 desenvolvimento de produto.

Na Figura 1 é apresentada a metodologia utilizada no desenvolvimento do CDA. $\mathrm{Na}$ cor laranja são demonstradas todas as fases do desenvolvimento do material pedagógico. As necessidades e as áreas envolvidas em cada fase estão em azul e, em verde, aspectos relevantes do processo.

É importante ressaltar no desenvolvimento do CDA a participação ativa dos membros do setor de EAD da universidade, que definiu com a equipe o recorte de conteúdos que seriam apresentados no CDA. Houve uma constante preocupação com a documentação do mesmo, pois, sendo uma equipe multidisciplinar, este serviu de guia para que todos seguissem os mesmos conceitos. 


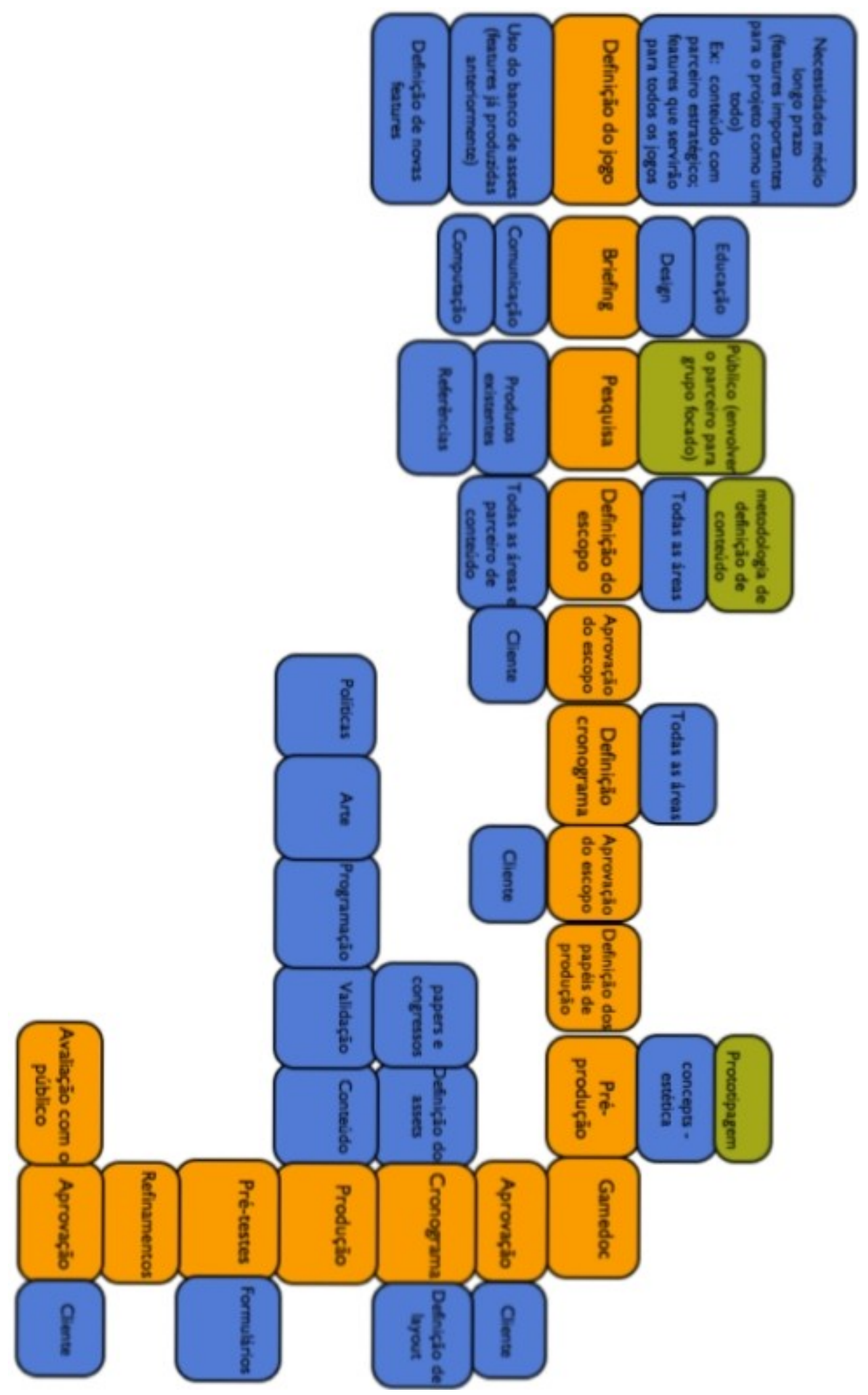

Figura 1 - Metodologia de desenvolVImento do COA "Introdução aos Estudos Virtuais•. (Bez et ai.,2012) 
Quanto à validação, esta ocorreu em dois momentos distintos: na blocagem, onde foi aplicada o método Think-Aloud (Erikson e Simon, 1993), (Xiao, 2000) e a aplicação de questionários aos participantes. Esta técnica consiste em observar usuários realizando tarefas e ações específicas, dentro de um ambiente controlado. Na realização das tarefas, deve-se estimular os participantes a verbalizarem as suas ações, informando o que estão fazendo e o resultado esperado para a ação que irão tomar (Villanueva, 2004). Com isso é possível perceber o que os mesmos estão observando e como estão se sentindo a cada interação realizada no sistema.

A segunda rodada de testes ocorreu com o CDA pronto e serviu para que fossem buscados aspectos a serem melhorados no mesmo. Pequenos erros, ajustes de performance e detalhes foram corrigidos.

\section{Desenvolvimento do CDA}

Seguindo a metodologia proposta, o CDA “Introdução aos Estudos Virtuais” foi desenvolvido. A definição do escopo ocorreu a partir de reuniões com os membros do projeto e o Núcleo de Educação a Distância (NEAD), discutindo as características da área. A definição do escopo e do cronograma foi discutida com todos os envolvidos, sendo apresentados em diversas reuniões ao NEAD para aprovação e definição dos papeis para a produção. Nesta fase, além do conteúdo, foram realizados estudos buscando referências para a estética e jogabilidade.

Foram definidos a prototipagem e os concepts do CDA na fase de pré-produção, estipulando-se que o mesmo deveria ser composto por missões e que, à medida que o aluno chegasse a determinado local, teria que responder a questões para seguir adiante no CDA (Figura 2).
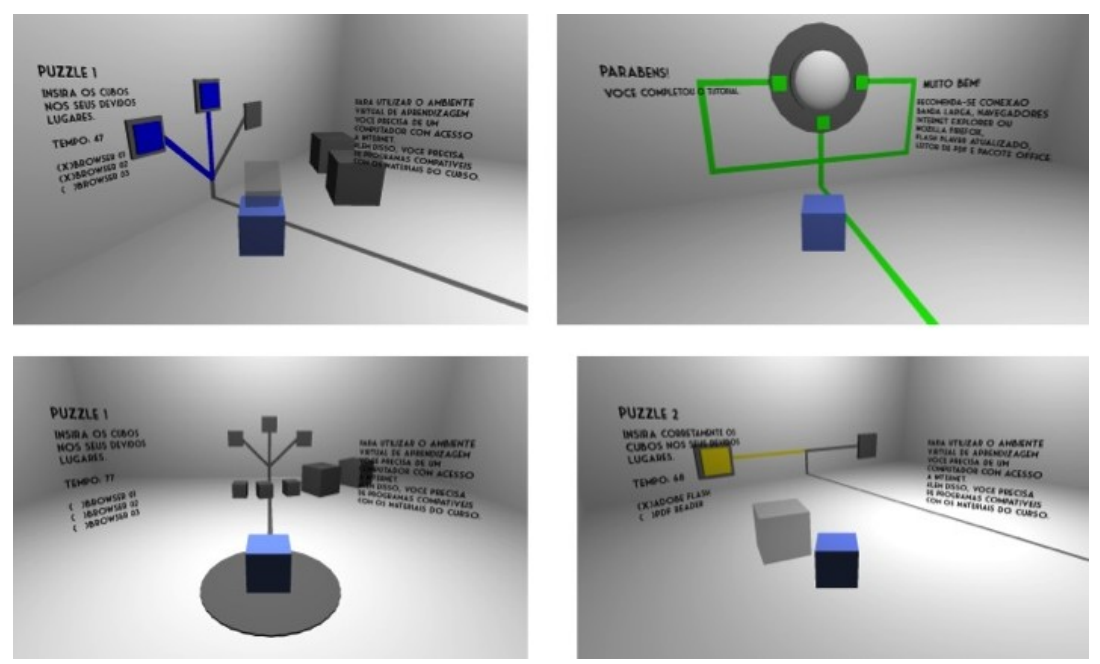

Figura 2. Protótipo na fase de pré-teste.

Foi criado um roteiro de missões que inseriram o conteúdo instrucional através da narrativa do ambiente. O universo de CDA ficou composto por cinco ilhas, cada uma contendo missões, tutoriais e filmes. A mecânica das missões foi dividida entre a busca por materiais pedagógicos sobre o tema, perguntas a serem respondidas e contatos com outros personagens do CDA, como, por exemplo, o professor, o tutor e os colegas. 
Após a definição das mecânicas básicas de jogabilidade que comporiam o CDA, assim como a maneira como o conteúdo seria apresentado ao usuário, criou-se um protótipo blocado do que viria a ser o construto. Este protótipo já contemplava o roteiro previamente estabelecido, assim como o fluxo de jogo definido durante as fases anteriores. Tanto o protótipo citado, quanto as demais versões do projeto foram construídos utilizando-se a ferramenta Unity 3D. Tendo tal estrutura pronta, foi realizada a primeira validação do jogo, na qual se convidou cinco pessoas, com idades que variaram de 16 até 64 anos, para testar o objeto desenvolvido até então. No local onde os testes foram realizados, instalou-se o CDA em um único computador, de forma que cada teste era feito de forma individual. Além disso, foram instaladas duas câmeras, uma para capturar a tela do computador e outra para registrar as reações do usuário (Figura 3), assim como um microfone para gravar seus comentários. Todos os experimentos foram acompanhados por ao menos um conhecedor do CDA para esclarecimento de dúvidas e um observador, que acompanhava as reações do usuário e anotava aspectos que considerava importantes. Todos usuários chegaram até o final do experimento, tendo-se registrado um tempo médio de 21 minutos para isso.

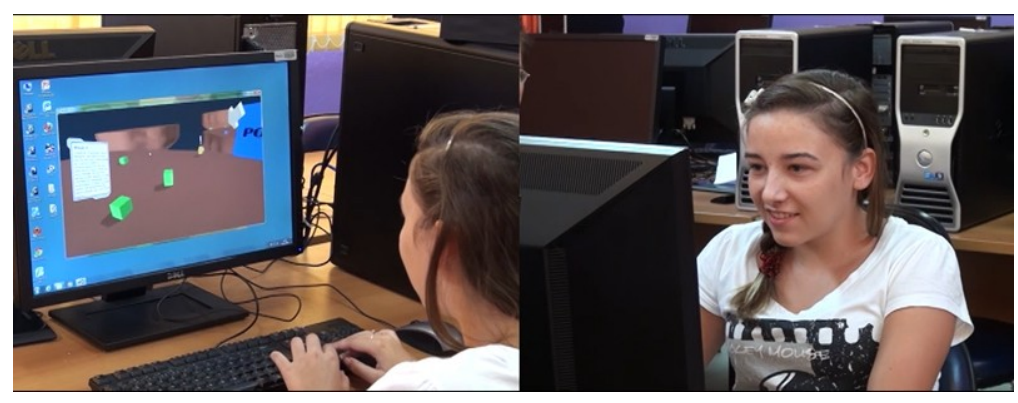

Figura 3. Resultado final do CDA.

Após a coleta de informações obtidas com a primeira validação, foram feitos diversos tipos de ajustes ao projeto, visando sempre sanar as dificuldades e críticas levantadas pelo grupo de teste. Foi também durante esta etapa que o projeto passou a contar com um aspecto mais acabado, já visando o que viria a ser o resultado final. Durante todo o período de produção, os representantes de cada área de conhecimento envolvida participaram de pelo menos uma reunião semanal do grupo. Ao final, obteve-se como resultado o CDA apresentado na Figura 4.

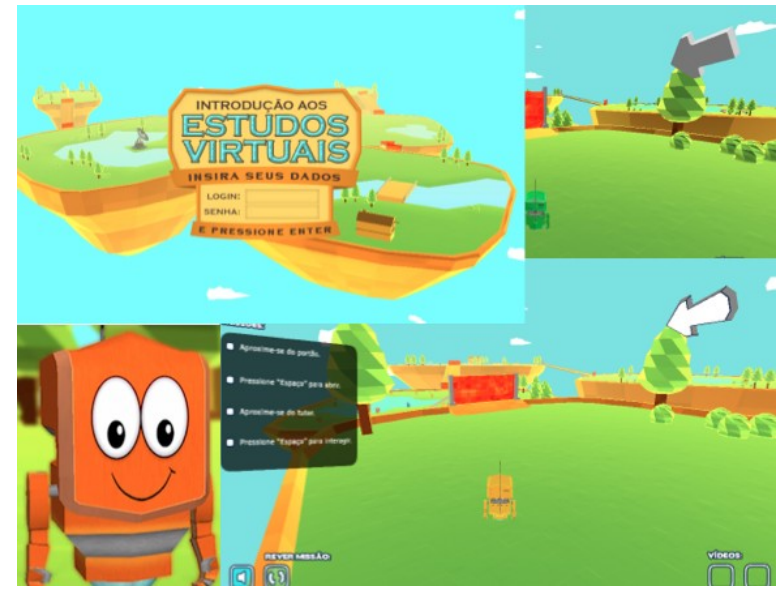

Figura 4. Resultado final do CDA. 
Uma segunda fase de validação ocorreu após dois meses da primeira, já com o produto pronto. $\mathrm{O}$ formato foi o mesmo da primeira, usando-se novamente o método de Think-Aloud, com os mesmos usuários e com duas câmeras e um microfone. O objetivo desta validação foi verificar se as dúvidas, necessidades e dificuldades apontadas pelos usuários na primeira fase de pré-testes, bem como as sugestões, haviam sido resolvidas na versão final do CDA e se este ainda apresentava algum tipo de inconsistência. A Figura 5 apresenta uma aluna testando o CDA final.

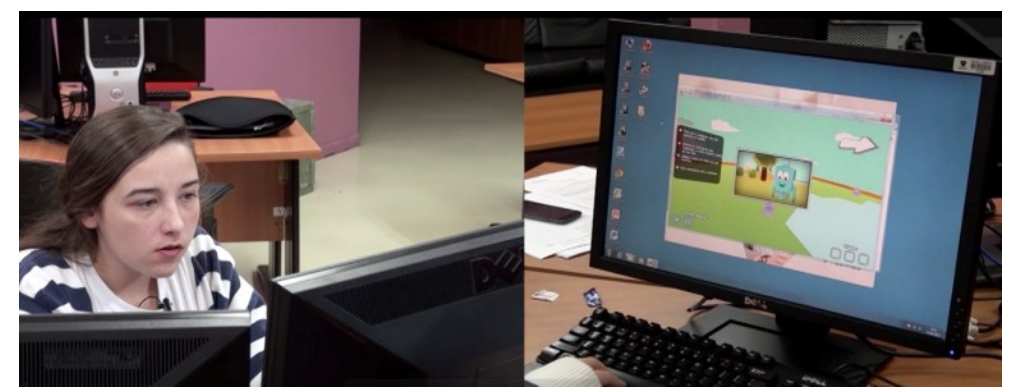

Figura 5. Validação final do CDA.

Na próxima seção são apresentados os resultados de uso do CDA “Introdução aos Estudos Virtuais” em dois semestres na instituição.

\section{Uso do CDA}

O CDA “Introdução aos Estudos Virtuais” foi disponibilizado aos alunos da Universidade Feevale no segundo semestre de 2013, no ambiente virtual de aprendizagem Blackboard, em uma tarefa obrigatória a todos os alunos de cursos totalmente a distância.

A tarefa na qual o construto foi aplicado tem o intuito de passar ao aluno todas as demandas e regras oriundas de um curso de EAD da instituição. Além disso, ela também pretende apresentar ao aluno um modelo de postura e netiqueta esperados dele para com os demais colegas de curso, tutores e professores no ambiente virtual.

Ao final do segundo semestre de 2013 constatou-se que 101 alunos acessaram o CDA no período dos cinco meses. No ambiente também foi aberto um fórum de discussão para que os alunos pudessem se expressar quanto à experiência de uso do CDA.

Ao total ocorreram 132 acessos ao CDA, por 101 estudantes. Alguns repetiram o experimento, executando o mesmo mais de uma vez. Dos estudantes que acessaram o CDA, 70 concluíram todas as tarefas, passando por todas as fases e 8 destes utilizaram o CDA até o final mais de uma vez. Do total de estudantes que utilizaram o construto, foi levantado que $62,5 \%$ eram do sexo masculino e $37,5 \%$ do sexo feminino.

O gráfico a seguir (figura 6) apresenta a distribuição por curso dos estudantes que utilizaram o CDA. Importante destacar que o somatório desta distribuição mostra um número diferente do total de estudantes que usaram o CDA, pois foram identificados alunos que participam em mais de um curso na instituição de ensino onde este fora utilizado. 


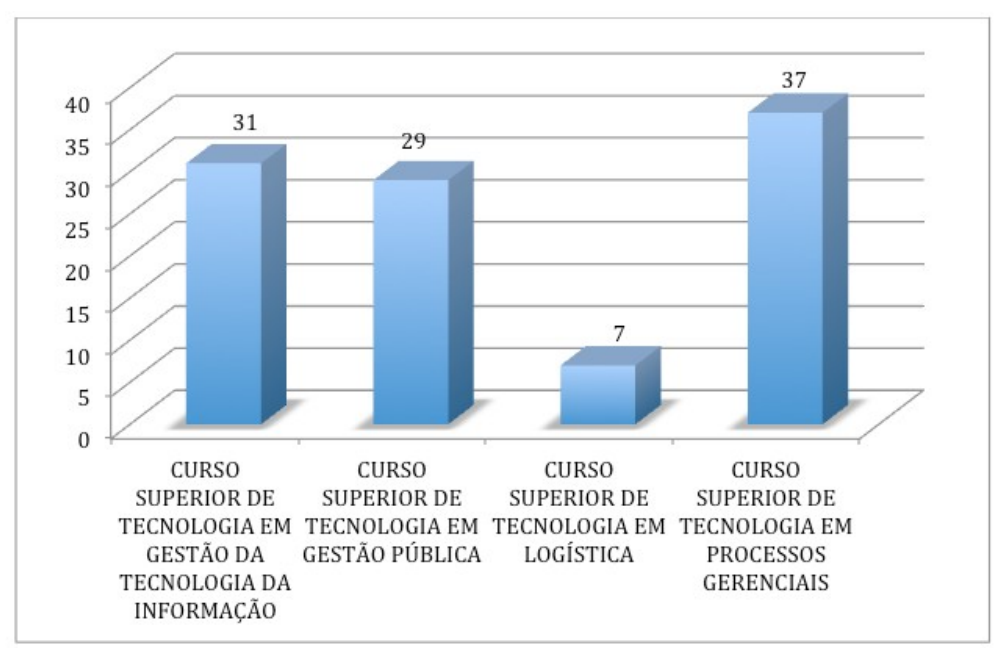

Figura 6. Quantidade de vezes que o CDA foi acessado por curso

O total de tempo acumulado durante todas as sessões deste experimento foi de 1.274 minutos. O maior tempo para término do CDA foi de 54 minutos e o menor de 12 minutos, sendo a média de 17 minutos por aluno. $\mathrm{O}$ aluno que demorou mais tempo para concluir o CDA (54 minutos) é do sexo masculino, tem 52 anos e estuda em dois cursos na instituição (Curso Superior de Tecnologia em Gestão Pública e Curso Superior de Logística). O aluno que concluiu em menor tempo (12 minutos) é do sexo masculino, tem 25 anos e estuda no curso Superior de Tecnologia em Logística. Ambos concluíram todas as etapas do construto.

Um fórum foi disponibilizado juntamente com a tarefa, permitindo aos alunos se expressarem quanto a aspectos positivos e negativos do CDA. Ao total, 55 interações foram realizadas no fórum, das quais duas críticas: "sem apelos para fixar o jogador” e "um pouco confuso", cada uma delas indicada por um estudante. As demais avaliações foram positivas, destacando-se os aspectos: "grande possibilidade de interação” (23 respostas), “objetivos claros" (10 respostas), "retrata o que é necessário para um aluno EAD” (20 respostas), "gentileza e cordialidade destacadas” (3 respostas), “didática simples e atrativa” (4 respostas), "lúdico, divertido e agradável” (8 respostas). Alguns alunos destacaram mais de um aspecto, o que faz com que número indicado seja maior do que o número de interações apresentadas.

Em termos de aprendizado com o CDA, os estudantes destacaram as seguintes palavras como necessárias para um aluno EAD: disciplina, iniciativa, organização, comprometimento e interação. Esses são os temas evidenciados no decorrer de todo o CDA, o que aponta que os estudantes conseguiram compreender e apreender o conteúdo repassado neste.

\section{Considerações finais}

Este artigo apresentou o trabalho realizado para o desenvolvimento do primeiro CDA feito pelo Laboratório de Objetos Aprendizagem da Universidade Feevale, assim como os resultados obtidos com a sua aplicação. O processo serviu de base para a elaboração de uma metodologia inicial de criação dos construtos digitais. 
A proposta descrita traz resultados que demonstram a eficácia da aplicação do CDA no grupo estudado e para o conteúdo em questão, sendo este as posturas e práticas nas disciplinas e cursos de EAD da instituição. No entanto, a metodologia de desenvolvimento deverá sofrer novas validações durante a produção de futuros CDAs.

Apenas com a sequência dos trabalhos e da aplicação em diferentes situações é que será possível dizer se tal teoria estará completamente validada, estabelecendo-se como um método a ser utilizado para a criação de construtos digitais de aprendizagem.

\section{Referencias Bibliográficas}

AN, Y.-J.; BONK, C. J. (2009) Finding that SPECIAL PLACE: Designing Digital Game-Based Learning. TechTrends, Berlin, v. 53, n. 3, p. 43-48, Maio / Junho 2009. ISSN 8756-3894.

BARAB, S.; PETTYJOHN, P.; GRESALFI, M.; VOLK, C.; SOLOMOU, M. (2011) Game-based curriculum and transformational play: Designing to meaningfully positioning person, content, and context. Computers \& Education 58; pp. 518-533

BECK, J. C., \& WADE, M. (2004). Got game. How the gamer generation is reshaping business forever. Boston, MA: Harvard Business School Press.

BEZ, M. R.; MOSSMANN, J. B.; MENDES, T. G.; BRANCO, M. (2012) Projeto para desenvolvimento de Objetos de Aprendizagem no formato de Jogos Sérios. In: Congreso Internacional de Ambientes Virtuales de Aprendizaje Adaptativos y Accesibles, 4., 2012, Cartagena. Anais do IV Congreso Internacional de Ambientes Virtuales de Aprendizaje Adaptativos y Accesibles, Cartagena: Cava, 2012, v.1, p. 1-10.

BOURGONJON, J.; VALCKE, M.; SOETAERT, R.; SCHELLENS, T. (2010) Student's perceptions about the use of video games in the classroom. Computers \& Education 54, pp. 1145-1156.

BOURGONJON, J.; RUTTEN, K.; VANHOOREN, S.; SOETAERT, R.. 2008. "Games Als \& in Educatie.” In Jongeren \& Gaming. Over De Effecten Van Games, Nieuwe Sociale Netwerken En Educatieve Kansen, ed. Evelien De Pauw, Stefaan Pleysier, Jan Van Looy, and Ronald Soetaert, 117-158.

BURGUILLO, Juan C. (2010) Using game theory and Competition-based Learning to stimulate student motivation and performance .Computers \& Education 55; pp. 566-575.

EBNER, M., \& HOLZINGER, A. (2007) Successful implementation of user-centered game based learning in higher education: an example from civil engineering. Computers \& Education 49; pp. 873-890.

ERICSSON, K.; SIMON, H. (1993) Protocol Analysis: Verbal Reports as Data. Cambridge, MA: The MIT Press.

GARRIS, R.; AHLERS, R.; DRISKELL, J. E. Games, motivation and learning. Simulation \& Gaming; An Interdisciplinary Journal of Theory, Practive and Research, Nice, v. 33, n. 4, p. 441-467, Dezembro 2002. ISSN 1046-8781 
GEE, J. P. (2003). What video games have to teach us about learning. New York: Palgrave.

LAWRENCE, R. (2004). Teaching data structures using competitive games. IEEE Transactions on Education, 47(4), pp.459-466.

MORAIS, A. M. Tomada de Decisão aplicada à Inteligência Artificial em Serious Games voltados para Saúde. In: Encontro Regional de Matemática Aplicada e Computacional ERMAC, 9., 2009, João Pessoa. Anais do IX Encontro Regional de Matemática Aplicada e Computacional - IX ERMAC, João Pessoa: SBMAC, 2009, 11p. [CD-Rom].

NMC. Horizon Project Short List: 2013 Higher Education Edition. Disponível em: $<$ http://www.nmc.org/pdf/-horizon-higher-ed-shortlist.pdf $>$. Acesso em dezembro de 2013.

OBLINGER D. G., \& OBLINGER, J. L. (2005). Educating the net generation. Online e-book: Educause.

PAPERT, S. (1980). Mindstorms: Children computers and powerful ideas. New York: Basic Books.

PILLAY, H. (2002). An investigation of cognitive processes engaged in by recreational computer game players: implications for skills of the future. Journal of Research on PPIG'05. pp. 293-304.

PRENSKY, M. (2001). Digital natives, digital immigrants, Part II: Do they really think differently? On the Horizon, 9(6), pp. 1-9.

RIBEIRO, P., SIMÕES, H., \& FERREIRA, M. (2009). Teaching artificial intelligence and logic programming in a competitive environment. Informatics in Education, 8(1), pp.85-100.

RIEBER, L. P. (1996). Seriously considering play: Designing interactive learning environments based on the blending of microworlds, simulations, and games. Educational Technology Research \& Development, 44(2), pp. 43-58.

RUSHKOFF, D. (1997). Ecstasy club. San Francisco: Harper San Francisco.SLAVIN, R. E. (1980). Cooperative learning. Review of Educational Research, 50(2), pp.315-342.

SCHULMEISTER, R. (2008). Is there a net gener in the house dispelling a mystification. E-Learning \& Education.

VILLANUEVA, R. A. (2004) Think-aloud protocol aril heuristic evaluation of non-immersive, desktop photo-realistic virtual environments.

WALLACE, S. A., \& MARGOLIS, J. (2007). Exploring the use of competitive programming: observations from the classroom. Journal of Computing in Small Colleges, 23(2), pp.33-39.

WATSON, W. (2007). Formative research on an instructional design theory for educational video games. Unpublished doctoral dissertation, Indiana University.

XIAO, D. Y. Experiencing the library in a panorama virtual reality environment. 2000. 